\title{
Anticoagulant effect of low concentration plasma trisodium citrate in continuous veno-venous hemofiltration
}

\author{
Jie Long ${ }^{1 *}$, Zhanting $\mathrm{Li}^{1 *}$, Ziyu Zhang ${ }^{1}$, Xuhui Dong ${ }^{1}$, Changliang Zhu $^{2}$ \\ ${ }^{1}$ Department of Nephrology, Xi'an International Medical Center Hospital, Xi'an, China; ${ }^{2}$ Department of Intensive Care Unit, Honghui Hospital, \\ Xi'an Jiaotong University, Xi'an, China \\ Contributions: (I) Conception and design: J Long, C Zhu; (II) Administrative support: Z Li; (III) Provision of study materials or patients: Z Zhang; \\ (IV) Collection and assembly of data: X Dong; (V) Data analysis and interpretation: J Long, C Zhu; (VI) Manuscript writing: All authors; (VII) Final \\ approval of manuscript: All authors. \\ "These authors contributed equally to this work. \\ Correspondence to: Changliang Zhu. Department of Intensive Care Unit, Honghui Hospital, Xi'an Jiaotong University, Xi'an 710054, China. \\ Email: piggyzc1926@163.com.
}

Background Effective anticoagulation is a prerequisite of continuous renal replacement therapy (CRRT). During CRRT, prolonged anticoagulant therapy should be administered to prevent extracorporeal clotting. The purpose of our study was to evaluate whether a low concentration of plasma trisodium citrate (TCA) was effective as an anticoagulation treatment for continuous veno-venous hemofiltration (CVVH). We also retrospectively analyzed the complications of regional citrate anticoagulation (RCA) in our hospital.

Methods: We conducted this single-center retrospective study at a tertiary academic hospital. The study period was from July 1, 2019 to January 1, 2021. CVVH using the RCA protocol during the study period was performed. The RCA protocol and complications were included and analyzed. The concentrations of citrate in the blood and plasma were calculated.

Results: In the current study, we used low-dose TCA as an anticoagulant in $288 \mathrm{CVVH}$ runs for 78 patients. The median whole blood and plasma citrate concentrations were $2.13 \mathrm{mmol} / \mathrm{L}$ and $2.84 \mathrm{mmol} / \mathrm{L}$, respectively. Although only a low concentration of citrate was used, RCA achieved expected anticoagulation and ensured the life span of the hemofilter during CVVH therapy. Although hypocalcemia was common during RCA, serious consequences could be prevented by timely adjustment of the protocol. Citrate intoxication was rare in patients without relative contraindications for citric acid. We did not find RCAassociated hypernatremia.

Conclusions: We recommend the use of a low concentration of TCA $(2.5-3.0 \mathrm{mmol} / \mathrm{L}$ in plasma) in extracorporeal solution in the RCA protocol. Although RCA may cause some complications, serious complications can be prevented. Mild hypocalcemia and transient metabolic alkalosis can be corrected in a timely manner. We do not believe hypernatremia is a complication of RCA if the RCA protocol is appropriately applied.

Keywords: Citrate; concentration; anticoagulation; continuous renal replacement

Submitted Jun 02, 2021. Accepted for publication Jul 15, 2021.

doi: 10.21037/apm-21-1672

View this article at: https://dx.doi.org/10.21037/apm-21-1672 


\section{Introduction}

Effective anticoagulation is a prerequisite of continuous renal replacement therapy (CRRT). During CRRT, prolonged anticoagulant therapy should be administered to prevent extracorporeal clotting. Although heparin is the most commonly used anticoagulant in clinical practice, the drawbacks of heparin are well known (1). In the intensive care unit (ICU), patients may have contraindications to heparin treatment due to hemorrhage, hemorrhagic tendency, coagulopathy, or heparin-induced thrombocytopenia (1). An alternative method is regional citrate anticoagulation (RCA). RCA has been used as an alternative anticoagulant in intermittent hemodialysis and CRRT for several decades $(1,2)$.

Serum calcium is an essential factor in the coagulation process. Citric acid reduces the concentration of ionized calcium $\left(\mathrm{iCa}^{2+}\right)$ by chelating $\mathrm{iCa}^{2+}$ to achieve anticoagulation $(1,2)$. Citrate $\left(\mathrm{C}_{6} \mathrm{H}_{7} \mathrm{O}_{7}\right)$ is a small molecule with a molecular weight of 191 Daltons (2). During CRRT, the majority of citrate is removed by convection or diffusion $(3,4)$, while a minority of citrate is delivered in the body $(3,4)$. Citrate is involved in the Krebs cycle and is metabolized to bicarbonate (2). The effects and safety of RCA have been addressed in randomized controlled trials (5-10). During the standard RCA protocol, citrate is administered by pre-filter infusion (5-10). Citrate chelates $\mathrm{iCa}^{2+}$ and leads to hypocalcemia in the extracorporeal circuit (2). The anticoagulation effect is assessed by monitoring the level of pre-hemofilter $\mathrm{iCa}^{2+}(1,2)$. The target value of pre-filter $\mathrm{iCa}^{2+}$ is $<0.35 \mathrm{mmol} / \mathrm{L}(1,2)$. When the concentration of $\mathrm{iCa}^{2+}$ is $<0.25 \mathrm{mmol} / \mathrm{L}$, coagulation can be almost completely inhibited (2). Infusing calcium post-filter normalized the concentration of $\mathrm{iCa}^{2+}$ which can prevent system hypocalcemia and ensure safety (5-10). However, Schwarzer et al. reported that blood gas analysis was inaccurate for testing $\mathrm{iCa}^{2+}$ when the concentration of $\mathrm{iCa}^{2+}$ was in the low-level range. The error ranged up to $0.33(0.21-0.50)$ $\mathrm{mmol} / \mathrm{L}$ (11). This brings challenges to the traditional RCA protocol. Another recommended method is adjusting citrate infusion to achieve an approximate citrate concentration of 4-6 $\mathrm{mmol} / \mathrm{L}$ for effective anticoagulation (2). The determination of citrate is not a routine test in clinical practice. Citrate concentration can be calculated in the filter based on blood flow, pre-dilution fluid, and the dose of citrate. The concentration of citrate in the plasma can also be estimated. Compared with $\mathrm{iCa}^{2+}$ concentration, using the calculated citrate concentration in the filter as an anticoagulation index may be simple and effective for preventing inaccurate testing of $\mathrm{iCa}^{2+}$.

Although many studies on RCA in CRRT have been performed, in most of the studies, pre-filter $\mathrm{iCa}^{2+}$ was used as an anticoagulant index. Studies on calculated citrate concentration in the filter as an anticoagulant index are very limited (12). In our hospital, RCA is used as conventional anticoagulation. We used trisodium citrate (TCA) as the anticoagulant and achieved an approximately low citrate concentration extracorporeal circuit $(<4 \mathrm{mmol} / \mathrm{L})$. The purpose of our study was to evaluate whether a low concentration of plasma TCA is an effective anticoagulation treatment for continuous veno-venous hemofiltration (CVVH). We also retrospectively analyzed the complications of RCA in our hospital. We present the following article in accordance with the STROBE reporting checklist (available at https://dx.doi.org/10.21037/apm-21-1672).

\section{Methods}

\section{Study design}

We conducted this single-center retrospective study in Xi'an International Medical Center Hospital. From 2018, RCA has been used as a conventional anticoagulation protocol for CRRT. In our hospital, we keep records of CRRT therapy. Medical records of CRRT were used to collect information on CRRT and RCA protocols. The study period was from July 1, 2019 to January 1, 2021. Because CVVH was the most commonly used CRRT session in our hospital, our study design was aimed at CVVH. The study was approved by Xi'an International Medical Center Hospital ethics committee (2021010). The ethics committee of the hospital waived the need for written informed consent from the patients in our study. The Declaration of Helsinki (as revised in 2013) of the World Medical Association was followed in our study.

\section{Screening of patients and inclusion/exclusion criteria for patients}

All patients who received CRRT treatment in our hospital from July 1, 2019 to January 1, 2021 were screened. The inclusion criteria were as follows: (I) patients who received CVVH in our hospital during the study period; (II) duration of $\mathrm{CVVH} \geq 12$ hours; (III) age $>18$ years. The exclusion criteria were as follows: (I) patients $<18$ years; (II) duration of $\mathrm{CVVH}<12$ hours. 
Table 1 Dose-adjustment protocol for TCA and 5\% calcium chloride

\begin{tabular}{lc}
\hline Protocol & Speed $(\mathrm{mL} / \mathrm{h})$ \\
\hline $4 \%$ TCA & \\
Post-filter iCa ${ }^{2+}(\mathrm{mmol} / \mathrm{L})$ & \\
$<0.25$ & Decreased by $5 \mathrm{~mL} / \mathrm{h}$ \\
$0.25-0.35$ & No adjustment \\
$0.35-0.50$ & Increased by $5 \mathrm{~mL} / \mathrm{h}$ \\
$>0.50$ & Increased by $10 \mathrm{~mL} / \mathrm{h}$ \\
$5 \%$ calcium chloride & \\
Pre-filter iCa ${ }^{2+}(\mathrm{mmol} / \mathrm{L})$ & \\
$>1.40$ & Decreased by $4 \mathrm{~mL} / \mathrm{h}$ \\
$1.20-1.40$ & Decreased by $2 \mathrm{~mL} / \mathrm{h}$ \\
$1.0-1.20$ & No adjustment \\
$0.9-1.0$ & Increased by $2 \mathrm{~mL} / \mathrm{h}$ \\
$<0.9$ & Increased by $4 \mathrm{~mL} / \mathrm{h}$ \\
\hline
\end{tabular}

TCA, trisodium citrate; $\mathrm{iCa}^{2+}$, ionized calcium.

Table 2 Clinical characteristics of the 78 patients receiving CVVH $(\mathrm{n}=78)$

\begin{tabular}{lc}
\hline Characteristics & Value \\
\hline Age & $56.76 \pm 18.12$ \\
Male/female & $47 / 31$ \\
Vascular access & 69 \\
Femoral dialysis catheter & 9 \\
Internal jugular vein dialysis catheter & \\
Indication for CRRT & 10 \\
Uremia & 56 \\
Acute kidney injury & 4 \\
Volume overload & 5 \\
Severe heart failure & 3 \\
Others &
\end{tabular}

Serum creatinine before receiving CRRT $\quad 489.66 \pm 286.70$ $(\mu \mathrm{mol} / \mathrm{L})$

Hemoglobin ( $\mathrm{g} / \mathrm{L})$

$105.99 \pm 14.59$

Hematocrit (\%)

$33.37 \pm 4.57$

CVVH, continuous veno-venous hemofiltration; CRRT, continuous renal replacement therapy.
In our hospital, the following conditions were usually considered as contraindications of RCA: (I) TCA allergy; (II) uncorrected hypoxemia (arterial partial pressure of oxygen $<60 \mathrm{mmHg}$ ); (III) possible tissue hypoxia; (IV) hepatic failure; (V) intractable hypocalcemia. Anticoagulation was determined by physicians.

\section{CRRT protocol}

In our hospital, CRRT treatment was delivered using Fresenius multiFiltrate $\mathrm{Ci}-\mathrm{Ca}$ (Fresenius Medical Care AG \& CO, Germany). The hemofilters used in our hospital was Ultraflux AV1000S (membrane surface area $1.8 \mathrm{~m}^{2}$, Fresenius Medical Care AG \& CO, Germany). Vascular access was established using a dialysis catheter. Usually, the blood flow was set to $100-150 \mathrm{~mL} / \mathrm{min}$ during CRRT therapy. Predilution or a combination of pre-dilution and post-dilution was used in our hospital. The pre-hemofilter replacement fluid was infused at a rate of $1,000-3,000 \mathrm{~mL} / \mathrm{hour}$. The postdilution replacement fluid was $<25 \%$ of the plasma flow. In our hospital, CRRT included two modes: 12 and $\geq 24$ hours. All therapy protocols were determined by physicians (Table 1).

\section{RCA protocol}

In the CVVH protocol, 4\% (136 mmol/L) TCA (H20058912, Sichuan Nangeer, China) was pre-hemofilter infused separately. The initial dose of TCA was $150 \mathrm{~mL} / \mathrm{hour}$ for patients weighing $<50 \mathrm{~kg}, 170 \mathrm{~mL} /$ hour for patients weighing $50-60 \mathrm{~kg}, 180 \mathrm{~mL} /$ hour for patients weighing $60-70 \mathrm{~kg}$, and $190 \mathrm{~mL} / \mathrm{hour}$ for patients weighing $>70 \mathrm{~kg}$. We did not use the replacement solution provided by the manufacturer, but prepared replacement solution based on our formula. Because of the use of TCA, we decreased the concentration of sodium and bicarbonate in the replacement solution. The prescription of the replacement solution is presented in Table $\mathrm{S} 1$. The diluted $5 \%$ calcium chloride was continuously infused from the central vein.

During CVVH, electrolyte indexes were re-monitored every 4 hours. The target value of post-filter $\mathrm{iCa}^{2+}$ was $0.25-0.35 \mathrm{mmol} / \mathrm{L}$. We adjusted the doses of TCA and $5 \%$ calcium chloride according to the pre-filter and postfilter concentration of $\mathrm{iCa}^{2+}$ (Table 2). The physician could prescribe a $5 \%$ calcium chloride intravenous administration when pre-filter $\mathrm{iCa}^{2+}<0.9 \mathrm{mmol} / \mathrm{L}$.

A pre-filter sample was drawn from extracorporeal upstream of TCA and the replacement solution infusion. A post-filter sample was drawn from the venous chamber. 
Pre-filter samples were used to test electrolytes, $\mathrm{iCa}^{2+}$, and blood gas analysis. Post-filter samples were used to test $\mathrm{iCa}^{2+}$. Electrolytes were measured by the ion selective electrode method (Roche, Switzerland). Blood gas analysis and $\mathrm{iCa}^{2+}$ were measured by ABL80 (Radiometer Medical ApS, Denmark).

\section{Collection of variables}

All data were collected using electronic medical records and CRRT records. Baseline data included demographics, body mass index (BMI), laboratory variables, diagnosis, complications, comorbidities, length of stay in hospital, and outcomes. Data on CRRT included major causes of receiving CRRT, treatment time, frequency, CRRT models, blood flow, replacement solution, ultrafiltration rate, doses of TCA and $10 \%$ calcium gluconate, and life span of the hemofilter.

\section{Calculation of citrate concentration in the hemofilter}

The concentration of citrate in the filter was determined by the infusion of TCA, blood flow, and the pre-hemofilter replacement fluid. We calculated citrate concentration in blood and plasma.

The citrate concentration in blood was calculated as $(\mathrm{mmol} / \mathrm{L}): 136(\mathrm{mmol}) \times$ TCA $(\mathrm{mL} /$ hour $) /[$ blood flow $(\mathrm{mL} / \mathrm{min}) \times 60+$ pre-hemofilter replacement fluid (mL/hour) $+4 \%$ TCA (mL/hour)]

The citrate concentration in plasma was calculated as $(\mathrm{mmol} / \mathrm{L}): 136(\mathrm{mmol}) \times$ TCA $(\mathrm{mL} /$ hour$) /[$ blood flow $(\mathrm{mL} / \mathrm{min}) \times(1-$ hematocrit $) \times 60+$ pre-hemofilter replacement fluid (mL/hour) + 4\% TCA (mL/hour)].

\section{Complications of RCA}

In the course of treatment, all patients received routine electrocardiogram (ECG), blood pressure, respiratory, and blood oxygen saturation monitoring. Electronic medical records and laboratory variables were used for screening complications of RCA. We recorded potential complications of RCA including the following metabolic disorders: metabolic acidosis was defined when $\mathrm{pH}<7.35$ and base excess (BE) decreased $<-2$; metabolic alkalosis was defined when $\mathrm{pH}>7.45$ and $\mathrm{BE}$ increased $>+2$. We used $\mathrm{pH}$ and the ratio of total calcium $/ \mathrm{iCa}^{2+}$ to diagnose citric acid intoxication and citric acid overload. Citric acid intoxication was diagnosed if metabolic acidosis and the ratio of total calcium $/ \mathrm{iCa}^{2+}>2.5$ developed. When metabolic alkalosis complicated the ratio of total calcium $/ \mathrm{iCa}^{2+}>2.5$, citric acid overload was considered. Hypocalcemia was defined as serum $\mathrm{iCa}^{2+}<1.00 \mathrm{mmol} / \mathrm{L}$. Hypernatremia was defined if serum sodium $>145 \mathrm{mmol} / \mathrm{L}$. Both theoretical speculation and previous studies have indicated that RCA does not increase the risk of hemorrhage. An important reason for using RCA in hospital was to avoid aggravating hemorrhage. Therefore, we did not record hemorrhage as a complication of RCA.

\section{Statistical analysis}

We used Statistical Product and Service Solutions (SPSS) version 20.0 to conduct data analysis. Continuous variables with a normal distribution were presented as mean \pm standard deviation. Medians and quartiles were used to express continuous variables with a skewed distribution.

\section{Results}

From July 1, 2019 to January 1, 2021, 202 patients received renal replacement therapy. We screened those patients and included 78 patients according to the inclusion and exclusion criteria.

\section{Clinical characteristics of patients who received $C V V H$}

The clinical characteristics of patients who received CVVH in our hospital during the study period are presented in Table 2. Of these 78 patients, the mean age was 57 and the ratio of male/female was $48 / 31$. The indications for CRRT included uremia, acute kidney injury, and severe heart failure, among others. The mean hemoglobin and hematocrit were lower than the normal range because of hemorrhagic diseases and uremia.

\section{CVVH therapy and RCA protocol}

During the study period, $288 \mathrm{CVVH}$ therapies were performed. Of those, 171 (59.4\%) were performed in 12 hours, and 71 were $>72$ hours. The number of CVVH therapies of 1 patient spanned from 1 to 22 . The shortest and the longest life spans of the hemofilter were 8 and 54 hours, respectively. During CVVH, blood flow was set at $150-200 \mathrm{~mL} / \mathrm{min}$. The replacement fluid was infused pre-dilution or a combination of pre-dilution and postdilution. The median of 4\% citrate was 170 (P25: 165, P75: 
Table 3 CVVH therapy and RCA protocol $(\mathrm{n}=288)$

\begin{tabular}{|c|c|}
\hline $\mathrm{CVVH}$ & Value \\
\hline Duration of each CVVH & $12[12,24]$ \\
\hline 12 hours & 171 \\
\hline$\geq 72$ hours & 71 \\
\hline Longest CVVH treatment (hours) & 72 \\
\hline Number of treatments/patients & $1-22$ \\
\hline Life span of hemofilter (hours) & $12[12,24]$ \\
\hline \multicolumn{2}{|l|}{ Blood flow } \\
\hline $150 \mathrm{~mL} / \mathrm{min}$ & 213 \\
\hline $200 \mathrm{~mL} / \mathrm{min}$ & 75 \\
\hline \multicolumn{2}{|l|}{ Replacement solution } \\
\hline $2,000 \mathrm{~mL} /$ hour & 240 \\
\hline $3,000 \mathrm{~mL} / \mathrm{hour}$ & 48 \\
\hline \multicolumn{2}{|l|}{ Pre-dilution replacement solution } \\
\hline $1,000 \mathrm{~mL} /$ hour & 107 \\
\hline $2,000 \mathrm{~mL} /$ hour & 153 \\
\hline $3,000 \mathrm{~mL} / \mathrm{hour}$ & 28 \\
\hline 4\% TCA (mL/hour) & $170[165,180]$ \\
\hline Whole TCA concentration (mmol/L) & $2.13[1.87,2.19]$ \\
\hline Plasma TCA concentration (mmol/L) & $2.84[2.59,3.08]$ \\
\hline
\end{tabular}

$\mathrm{CVVH}$, continuous veno-venous hemofiltration; RCA, regional citrate anticoagulation; TCA, trisodium citrate.

Table 4 Complications of RCA ( $\mathrm{n}=288)$

\begin{tabular}{lc}
\hline Complications & Number \\
\hline Hypocalcemia (pre-hemofilter $\left.\mathrm{iCa}^{2+}<1.0 \mathrm{mmol} / \mathrm{L}\right)$ & 58 \\
Hypocalcemia (pre-hemofilter $\left.\mathrm{iCa}^{2+}<0.9 \mathrm{mmol} / \mathrm{L}\right)$ & 19 \\
Hypercalcemia (pre-hemofilter $\left.\mathrm{iCa}^{2+}>1.2 \mathrm{mmol} / \mathrm{L}\right)$ & 28 \\
Hypernatremia & 0 \\
Citrate accumulation & 1 \\
Metabolic alkalosis & 9 \\
Hemofilter clotting & 14 \\
\hline
\end{tabular}

$\mathrm{RCA}$, regional citrate anticoagulation; $\mathrm{iCa}^{2+}$, ionized calcium.

180) $\mathrm{mL} /$ hour. The median calculated concentrations of TCA in the whole blood and plasma were $2.13 \mathrm{mmol} / \mathrm{L}$ and $2.84 \mathrm{mmol} / \mathrm{L}$, respectively (Table 3).

\section{Complications of RCA}

The most common complication of RCA was hypocalcemia, however, this complication did not lead to serious consequences because of close monitoring and timely adjustment of calcium infusion. One patient with a severe infection was diagnosed with citrate intoxication based on metabolic acidosis and total $/ \mathrm{iCa}^{2+}$ ratio $>2.5$. RCA was stopped because of citrate intoxication. In the current study, we did not find RCA-associated hypernatremia. However, 14 patients ended the treatment or had their hemofilters replaced because of insufficient anticoagulation. Eleven patients were complicated with transient metabolic alkalosis ( $\mathrm{pH}>7.45$ and $\mathrm{BE}$ increased $>+2$ ) which was corrected by adjusting the base in the replacement solution (Table 4).

\section{Discussion}

In the current study, we used low-dose TCA as an anticoagulant for CVVH. The calculated citrate concentration was lower than recommended by Davenport and Tolwani (2). The median whole blood and plasma citrate concentrations were 2.13 and $2.84 \mathrm{mmol} / \mathrm{L}$, respectively. Although only low concentration citrate was used, RCA achieved expected anticoagulation and ensured the life span of the hemofilter during CVVH therapy. Although hypocalcemia was common during RCA, serious consequences could be prevented by timely adjustment of the protocol. Citrate intoxication was rare in patients without relative contraindications for citric acid. We did not find RCA-associated hypernatremia.

Effective anticoagulation is the prerequisite of CRRT. Different from intermittent hemodialysis treatment, CRRT treatment takes a long time and requires long-term anticoagulation (1). Long-term anticoagulation may be related to more complications. Although heparin is the most commonly used anticoagulant, its drawbacks are generally known. Especially in critical patients, heparin may aggravate bleeding $(1,13,14)$. In clinical practice, citrate and citric acid have been used as anticoagulants for many years $(1,2)$. Both acid citrate dextrose (ACD) and TCA are used for anticoagulation (2). Citrate chelates calcium and decreases the concentration of $\mathrm{iCa}^{2+}$, which is also a coagulation factor in the blood $(1,2)$. Calcium deficiency can achieve effective anticoagulation. Citric acid is involved in the Krebs cycle in the liver, muscle tissue, and renal cortex $(1,2)$. In healthy individuals, the half-life of citric acid is very short, around 5 minutes (2). As citric acid metabolizes rapidly in the body, 
it has no system anticoagulation in the body. Because of the potential advantages of RCA, RCA is a good anticoagulant for CRRT. Citrate has been widely used in CRRT $(1,2)$.

However, the disadvantages of RCA should be fully considered in clinical practice. The first point to be considered is the relatively complex protocol (5-10). Citrate should be infused pre-hemofilter and the concentration of $\mathrm{iCa}^{2+}$ should be reduced. Calcium-free replacement solution should be supplied. The compound of citric acid and calcium is small molecular and can be cleared by CRRT. The sieve coefficient is $0.87-1.0(3,4)$. Therefore, RCA decreases calcium in the blood, and extra $\mathrm{iCa}^{2+}$ should be supplied to prevent hypocalcemia. Usually, two methods are used to infuse citrate: citrate can be infused separately or used as a component of replacement fluid (15-17). In our hospital, we use 4\% TCA as an anticoagulant which is separately pre-hemofilter infused. Calcium-free replacement solution is also used. $\mathrm{iCa}^{2+}$ is delivered from the central vein. It should be noted that one molecule of TCA contains three molecules of sodium. Bicarbonate is produced by TCA metabolism in the body $(1,2)$. To prevent hypernatremia and metabolic alkalosis, we reduced the concentration of sodium bicarbonate in the replacement solution. According to the adjustment of RCA, the concentration of sodium bicarbonate in the replacement solution was adjusted in a timely manner. If TCA was increased $1 \mathrm{mmol} / \mathrm{hour}$, and the infusion of sodium bicarbonate in the replacement solution was reduced by $3 \mathrm{mmol} / \mathrm{L}$. In fact, TCA is not only an anticoagulant, but also a base in the replacement solution. When the complications of RCA are considered, hypernatremia and metabolic alkalosis are often mentioned. However, if TCA is also regarded as a base and the supplier of sodium ions in the replacement solution, timely adjustment of other components in the replacement solution according to the dose of TCA can prevent hypernatremia.

Usually, patients with renal failure are prone to metabolic acidosis, so conventional dialysate and replacement solutions contain a higher level of base than the normal level. With CRRT therapy and the correction of acidosis, the concentration of base should be adjusted in a timely manner to prevent metabolic alkalosis. In the current study, we did not find hypernatremia and persistent metabolic alkalosis induced by RCA. We believe that hypernatremia and metabolic alkalosis can be prevented if the RCA protocol is appropriately applied. CRRT can also regulate acid-base disorders and electrolyte disorders (18). Therefore, our choice is infusing TCA separately and adjusting the prescription of replacement solution in a timely manner according to the patient's condition, which may be beneficial for patients.

When designing the protocols for CRRT and RCA, the first thing that should be considered is the dose of TCA. Based on previous research, the dose of TCA ranges from 17 to $45 \mathrm{mmol} / \mathrm{h}(15,16,19)$. However, if TCA is thought of as a base and the supplier of sodium ions, high-dose TCA is not suitable for treatment when the replacement solution is low. Normally, the sodium ions in the replacement solution are supplied from sodium chloride and sodium bicarbonate. Sodium in the replacement solution should be reduced when TCA is supplied. Because $1 \mathrm{mmol}$ TCA contains $3 \mathrm{mmol}$ sodium, $45 \mathrm{mmol} / \mathrm{hour}$ TCA metabolizes $135 \mathrm{mmol}$ sodium/hour. A significant reduction in the sodium concentration of the replacement solution will lead to hypochloremia. At present, not enough evidence supports the application of high-dose replacement fluid to reduce mortality (20). Usually, in our hospital, the replacement fluid volume is $2,000 \mathrm{~mL} /$ hour. Therefore, high-dose TCA is not our choice for the RCA protocol. The initial dose of TCA is $150-190 \mathrm{~mL} 4 \%$ TCA/hour (20.4-25.8 $\mathrm{mmol} / \mathrm{h})$. In the current study, the highest dose of TCA was $220 \mathrm{~mL} \mathrm{4 \%} \mathrm{TCA/hour}(29.9 \mathrm{mmol} / \mathrm{h})$. Some previous studies also support the use of low-dose citrate for CRRT anticoagulation (21-23).

Effective RCA depends on the decrease of $\mathrm{iCa}^{2+}(1,2)$. When the concentration of $\mathrm{iCa}^{2+}$ is $>0.50 \mathrm{mmol} / \mathrm{L}$, almost no anticoagulation is achieved $(6,24)$. When the calcium concentration is $<0.25 \mathrm{mmol} / \mathrm{L}$, coagulation cascades are almost completely inhibited (2). Therefore, anticoagulation can be assessed by monitoring the extracorporeal $\mathrm{iCa}^{2+}$ concentration (the target value is $0.25-0.30 \mathrm{mmol} / \mathrm{L}$ of $\mathrm{iCa}^{2+}$ concentration) (2). In the current study, we used a relatively low dose of TCA, and the target value was $0.25-0.35 \mathrm{mmol} / \mathrm{L}$ of $\mathrm{iCa}^{2+}$ concentration (6). In most cases, effective anticoagulation was achieved. However, in two cases, the life span of the hemofilter was only 8 hours. Although $\mathrm{iCa}^{2+}$ is the most common indicator for RCA, it does not seem to be as reliable as we think. Schwarzer et al. indicated that blood gas analysis is not an ideal method to test $\mathrm{iCa}^{2+}$. When $\mathrm{iCa}^{2+}$ concentration is at a low level, blood gas analysis is inaccurate for $\mathrm{iCa}^{2+}$ determination (11). The error of the result ranged as high as $0.33(0.21-0.50) \mathrm{mmol} / \mathrm{L}$. This may lead to inaccurate adjustment of the RCA protocol. Previous studies have indicated that a concentration of $4-6 \mathrm{mmol} / \mathrm{L}$ citrate can reduce the $\mathrm{iCa}^{2+}$ to $<0.2 \mathrm{mmol} / \mathrm{L}$ and prevent activation of both coagulation and platelets. Therefore, Davenport 
and Tolwani recommended to adjust the dose of citrate according to blood flow, to achieve an approximate citrate concentration of 4-6 $\mathrm{mmol} / \mathrm{L}$ for effective anticoagulation (2). In the current study, we calculated the concentration of TCA in the whole blood and estimated the concentration of TCA in the plasma. We found that a relatively low concentration of TCA can also achieve effective anticoagulation. The estimated concentration of TCA in plasma ranged from $2.59-3.08 \mathrm{mmol} / \mathrm{L}$. The results of the current study indicate that the citrate concentration of $2.6-3.0 \mathrm{mmol} / \mathrm{L}$ results in effective anticoagulation. The TCA dosage, blood flow, and pre-dilution replacement solution can be adjusted to achieve the target value of citrate concentration. Combining system $\mathrm{iCa}^{2+}$ monitoring and estimating the TCA concentration in the hemofilter is effective and simple.

Anticoagulation in CRRT is a complex process. In addition to anticoagulants, many factors such as abnormality of the coagulation-anticoagulation system, blood flow, predilution or post-dilution replacement solutions, dialysis catheter, hemoglobin, platelets, and other factors may affect anticoagulation (1). In the current study, we used a low concentration of TCA as an anticoagulation treatment, and the protocol also achieved the expected anticoagulation (1). The following aspects of this study might have contributed to improving the anticoagulation effect: (I) we used predilution or a combination of pre-dilution and postdilution to deliver the replacement solution. Pre-dilution can prevent hemoconcentration and decrease the $\mathrm{iCa}^{2+}$ concentration in the hemofilter; (II) for most treatments, the CRRT session only lasted for 12 hours; (III) the mean hemoglobin level was lower than the normal level.

The complications of RCA include hypocalcemia, acidbase disorders, hypernatremia, or hypercalcemia $(1,2,25)$. Sodium citrate metabolism in vivo is as follows: $\mathrm{Na}_{3}$ citrate $+3 \mathrm{H}_{2} \mathrm{HCO}_{3} \rightarrow$ citric acid $\left(\mathrm{C}_{6} \mathrm{H}_{8} \mathrm{O}_{7}\right)+3 \mathrm{NaHCO}_{3}$. Blood $\mathrm{pH}$ value is mainly determined by three variables: $\mathrm{PaCO}_{2}$, strong ion difference (SID), and weak acid concentration. Citric acid is a weak acid which can lead to liquid acidification $(1,2)$. However, if TCA is infused, the increase of SID will cause alkalinity. Sodium citrate may cause acid-base disorders when it is delivered. If too much sodium citrate is given and TCA metabolizes to produce redundant bicarbonate in the body, it may cause metabolic alkalosis (14). If the metabolic pathway of citric acid in the body is damaged, accumulation of citric acid in the body may lead to citric acid intoxication, which presents as metabolic acidosis and total serum calcium $/ \mathrm{Ca}^{2+}>2.5$ (14).
In the current study and previous studies, metabolic alkalosis induced by RCA was reported. However, in most cases, metabolic alkalosis was mild and transient. Timely adjustment can correct the metabolic alkalosis. Citric acid intoxication may be a serious complication of RCA. When citric acid cannot be normally metabolized, citric acid intoxication leads to severe hypocalcemia and metabolic acidosis. Identifying contraindications of RCA is of great importance. Although previously liver failure was considered a contraindication of RCA, a few studies have supported the use of RCA in patients with liver failure (26). However, the results need further exploration. Citrate metabolism depends on the Krebs cycle which requires consumption of oxygen. Therefore, hypoxemia, especially tissue hypoxia, may cause severe complications of RCA (2).

Finally, we refer to the advantages and disadvantages of our study. An ideal anticoagulant therapy should reduce the dosage of anticoagulant as much as possible on the premise of effective anticoagulation (2). Although previous studies have recommended achieving an approximate citrate concentration of $4-6 \mathrm{mmol} / \mathrm{L}$ for effective anticoagulation (2), our results support the use of a lower concentration of TCA in the RCA protocol. Considering that no evidence strongly supports the application of highdose replacement solution, we preferred 12-hour CRRT rather than 24-hour CRRT in most cases. Therefore, we believe that a low concentration of TCA is enough for anticoagulation. When RCA is used as anticoagulation, an important tip is to fully consider the proficiency of medical staff. We do not believe hypernatremia is a complication of RCA if the RCA protocol is appropriately applied. Mild hypocalcemia and transient metabolic alkalosis can also be corrected in a timely manner to prevent serious consequences. We recommend the use of a low concentration of TCA in extracorporeal solution in the RCA protocol. The results should be further confirmed by a randomized controlled study, as single-center retrospective studies are unpersuasive. Anticoagulation is a complex process, and we did not include all factors contributing to the coagulation-anticoagulation system. This is also a limitation of our study. Another important limitation is that we did not measure the actual citrate concentration.

\section{Conclusions}

We recommend the use of a low concentration of TCA (2.5-3.0 $\mathrm{mmol} / \mathrm{L}$ in plasma) in extracorporeal solution in the RCA protocol. Although RCA may cause some 
complications, serious complications can be prevented. Mild hypocalcemia and transient metabolic alkalosis can be corrected in a timely manner. We do not believe that hypernatremia is a complication of RCA if the RCA protocol is appropriately applied.

\section{Acknowledgments}

Funding: None.

\section{Footnote}

Reporting Checklist: The authors have completed the STROBE reporting checklist. Available at https://dx.doi. org/10.21037/apm-21-1672

Data Sharing Statement: Available at https://dx.doi. org/10.21037/apm-21-1672

Conflicts of Interest: All authors have completed the ICMJE uniform disclosure form (available at https://dx.doi. org/10.21037/apm-21-1672). The authors have no conflicts of interest to declare.

Ethical Statement: The authors are accountable for all aspects of the work in ensuring that questions related to the accuracy or integrity of any part of the work are appropriately investigated and resolved. The study was approved by Xi'an International Medical Center Hospital ethics committee (2021010). The ethics committee of the hospital waived the need for written informed consent from the patients in our study. The Declaration of Helsinki (as revised in 2013) of the World Medical Association was followed in our study.

Open Access Statement: This is an Open Access article distributed in accordance with the Creative Commons Attribution-NonCommercial-NoDerivs 4.0 International License (CC BY-NC-ND 4.0), which permits the noncommercial replication and distribution of the article with the strict proviso that no changes or edits are made and the original work is properly cited (including links to both the formal publication through the relevant DOI and the license). See: https://creativecommons.org/licenses/by-nc-nd/4.0/.

\section{References}

1. Oudemans-van Straaten HM, Wester JPJ, de Pont A, et al.
Anticoagulation strategies in continuous renal replacement therapy: can the choice be evidence based? Intensive Care Med 2006;32:188-202.

2. Davenport A, Tolwani A. Citrate anticoagulation for continuous renal replacement therapy (CRRT) in patients with acute kidney injury admitted to the intensive care unit. NDT Plus 2009;2:439-47.

3. Chadha V, Garg U, Warady BA, et al. Citrate clearance in children receiving continuous venovenous renal replacement therapy. Pediatr Nephrol 2002;17:819-24.

4. Swartz R, Pasko D, O'Toole J, et al. Improving the delivery of continuous renal replacement therapy using regional citrate anticoagulation. Clin Nephrol 2004;61:134-43.

5. Monchi M, Berghmans D, Ledoux D, et al. Citrate vs. heparin for anticoagulation in continuous venovenous hemofiltration: a prospective randomized study. Intensive Care Med 2004;30:260-5.

6. Kutsogiannis DJ, Gibney RT, Stollery D, et al. Regional citrate versus systemic heparin anticoagulation for continuous renal replacement in critically ill patients. Kidney Int 2005;67:2361-7.

7. Betjes MG, van Oosterom D, van Agteren M, et al. Regional citrate versus heparin anticoagulation during venovenous hemofiltration in patients at low risk for bleeding: similar hemofilter survival but significantly less bleeding. J Nephrol 2007;20:602-8.

8. Fealy N, Baldwin I, Johnstone M, et al. A pilot randomized controlled crossover study comparing regional heparinization to regional citrate anticoagulation for continuous venovenous hemofiltration. Int J Artif Organs 2007;30:301-7.

9. Oudemans-van Straaten HM, Bosman RJ, Koopmans M, et al. Citrate anticoagulation for continuous venovenous hemofiltration. Crit Care Med 2009;37:545-52.

10. Hetzel GR, Schmitz M, Wissing H, et al. Regional citrate versus systemic heparin for anticoagulation in critically ill patients on continuous venovenous haemofiltration: a prospective randomized multicentre trial. Nephrol Dial Transplant 2011;26:232-9.

11. Schwarzer P, Kuhn SO, Stracke S, et al. Discrepant post filter ionized calcium concentrations by common blood gas analyzers in CRRT using regional citrate anticoagulation. Crit Care 2015;19:321.

12. Cointault O, Kamar N, Bories P, et al. Regional citrate anticoagulation in continuous venovenous haemodiafiltration using commercial solutions. Nephrol Dial Transplant 2004;19:171-8.

13. Bai $M$, Zhou $M$, He L, et al. Citrate versus heparin 
anticoagulation for continuous renal replacement therapy: an updated meta-analysis of RCTs. Intensive Care Med 2015;41:2098-110.

14. Schneider AG, Journois D, Rimmele T. Complications of regional citrate anticoagulation: accumulation or overload? Crit Care 2017;21:281.

15. Schilder L, Nurmohamed SA, Bosch FH, et al. Citrate anticoagulation versus systemic heparinisation in continuous venovenous hemofiltration in critically ill patients with acute kidney injury: a multi-center randomized clinical trial. Crit Care 2014;18:472.

16. Stucker F, Ponte B, Tataw J, et al. Efficacy and safety of citrate-based anticoagulation compared to heparin in patients with acute kidney injury requiring continuous renal replacement therapy: a randomized controlled trial. Crit Care 2015;19:91.

17. Palsson R, Niles JL. Regional citrate anticoagulation in continuous venovenous hemofiltration in critically ill patients with a high risk of bleeding. Kidney Int 1999;55:1991-7.

18. Yessayan L, Yee J, Frinak S, et al. Continuous renal replacement therapy for the management of acid-base and electrolyte imbalances in acute kidney injury. Adv Chronic Kidney Dis 2016;23:203-10.

19. Gattas DJ, Rajbhandari D, Bradford C, et al. A randomized controlled trial of regional citrate versus regional heparin anticoagulation for continuous renal replacement therapy in critically ill adults. Crit Care Med 2015;43:1622-9.

20. Wu B, Ji D, Xu B, et al. New modes of continuous renal

Cite this article as: Long J, Li Z, Zhang Z, Dong X, Zhu C. Anticoagulant effect of low concentration plasma trisodium citrate in continuous veno-venous hemofiltration. Ann Palliat Med 2021;10(8):8900-8908. doi: 10.21037/apm-21-1672 replacement therapy using a refiltering technique to reduce micronutrient loss. Hemodial Int 2019;23:181-8.

21. Egi M, Naka T, Bellomo R, et al. The acid-base effect of changing citrate solution for regional anticoagulation during continuous veno-venous hemofiltration. Int J Artif Organs 2008;31:228-36.

22. Thoenen M, Schmid ER, Binswanger U, et al. Regional citrate anticoagulation using a citrate-based substitution solution for continuous venovenous hemofiltration in cardiac surgery patients. Wien Klin Wochenschr 2002;114:108-14.

23. Naka T, Egi M, Bellomo R, et al. Low-dose citrate continuous veno-venous hemofiltration $(\mathrm{CVVH})$ and acidbase balance. Int J Artif Organs 2005;28:222-8.

24. Oudemans-van Straaten HM, Ostermann M. Citrate anticoagulation for CRRT: don't always trust the postfilter iCa results! Crit Care 2015;19:429.

25. Brain $\mathrm{M}$, Winson E, Roodenburg O, et al. Non anticoagulant factors associated with filter life in continuous renal replacement therapy (CRRT): a systematic review and meta-analysis. BMC Nephrol 2017;18:69.

26. Ma Y, Chen F, Xu Y, et al. Safety and efficacy of regional citrate anticoagulation during plasma adsorption plus plasma exchange therapy for patients with acuteon-chronic liver failure: a pilot study. Blood Purif 2019;48:223-32.

(English Language Editor: B. Draper) 
Supplementary

Table S1 The prescription of the replacement solution

\begin{tabular}{lcc}
\hline \multirow{2}{*}{ Component } & \multicolumn{2}{c}{ Labelled amount } \\
\cline { 2 - 3 } & Value 1 (mmol/L) & Value 2 $(\mathrm{mg} / \mathrm{mL})$ \\
\hline $\mathrm{C}_{6} \mathrm{H}_{12} \mathrm{O}_{6}$ & 10.6 & 1.91 \\
$\mathrm{Cl}$ & 118 & 4.18 \\
$\mathrm{Mg}$ & 0.797 & 0.0194 \\
$\mathrm{Ca}$ & 1.60 & 0.0639 \\
$\mathrm{Na}$ & 113 & 2.60 \\
\hline
\end{tabular}

